\title{
Aspects of topological actions on the lattice
}

\section{Oscar Åkerlund}

Institut für Theoretische Physik, ETH Zurich, CH-8093 Zürich, Switzerland

E-mail: oscara@phys.ethz.ch

\section{Philippe de Forcrand*}

Institut für Theoretische Physik, ETH Zurich, CH-8093 Zürich, Switzerland CERN, Physics Department, TH Unit, CH-1211 Geneva 23, Switzerland

E-mail: forcrandephys.ethz.ch

\begin{abstract}
We consider a lattice action which forbids large fields, and which remains invariant under smooth deformations of the field. Such a "topological" action depends on one parameter, the field cutoff, but does not have a classical continuum limit as this cutoff approaches zero. We study the properties of such an action in $4 d$ compact $U(1)$ lattice gauge theory, and compare them with those of the Wilson action. In both cases, we find a weakly first-order transition separating a confining phase where monopoles condense, and a Coulomb phase where monopoles are exponentially suppressed. We also find a different, critical value of the field cutoff where monopoles completely disappear. Finally, we show that a topological action simplifies the measurement of the free energy.
\end{abstract}

The 33rd International Symposium on Lattice Field Theory

14 -18 July 2015

Kobe International Conference Center, Kobe, Japan

\footnotetext{
*Speaker.
} 


\section{Introduction}

At least two strategies can be adopted to improve the approach of a lattice action to the continuum limit. The most widely considered is the Symanzik strategy [1], where irrelevant operators of increasing dimension are added to the standard action, with coefficients determined perturbatively or not. Another, less common, strategy is motivated by the observation that the approach to the continuum behavior is faster for weaker fields: suppressing strong fields will improve the action in a non-parametric way. Indeed, the Manton action [2], or renormalization-group improved actions like the "perfect" action [3] all suppress large fields.

A radical implementation of this second strategy consists of imposing a cutoff on the amount of local excitation, the earliest example being the positive-plaquette action [4]. Recently, a combination of Wilson-type action and cutoff constraint has been shown to display much improved scaling in a spin model [5]. Very recently, a similar approach has been applied to a gauge theory [6].

This leaves open the question of the approach to the continuum limit of a constraint-only action, namely an action $S$ such that $\exp (-S)=1$ if every local constraint is satisfied, 0 otherwise. Such an action, which is invariant under small deformations of the field, is for this reason called "topological". While it is intuitively clear that the correlation length will keep increasing as the constraint selects weaker and weaker fields, it is not at all obvious which continuum theory will be approached: a gradient expansion of the lattice action is not possible, since the action is either zero or infinite. Nevertheless, it has been shown numerically [7] that in a $2 d X Y$ spin model, the same Coulomb phase is obtained as with the standard action. Here, we study the case of a $4 d U(1)$ gauge theory, and compare the Wilson action $S_{W}=-\beta \sum_{P} \cos \theta_{P}$ with the topological action

$$
\exp \left(-S_{\text {top }}\right)=\prod_{P} \Theta\left(\delta-\left|\theta_{P}\right|\right)
$$

where $\theta_{P}$ is the angle of plaquette $P$ and $\delta \in[0, \pi]$ is the cutoff on $\theta_{P}$. Most of the results presented here have appeared in [8].

\section{Monopoles and ergodicity}

It is well-known that, using the Wilson action, the $4 d U(1)$ lattice gauge theory has two phases: Coulomb at weak coupling, confining at strong coupling, with a weak first-order transition at $\beta_{c} \approx$ $1.01 \cdots$. Moreover, in the confining phase magnetic monopoles condense [9]. With a topological action, the existence of monopoles is governed by the cutoff angle $\delta$. As shown Fig. 1 (left), a total flux of $2 \pi$ through the 6 faces of a cube, which characterizes a monopole, requires $\delta$ to be at least $\pi / 3$. Thus the monopole density vanishes as $\delta \rightarrow \frac{\pi}{3}^{+}$, and is strictly zero for $\delta<\frac{\pi}{3}$. This non-analyticity of the monopole density causes a phase transition at $\delta=\pi / 3$, which one may guess coincides with the Coulomb-confining transition. This guess is wrong as we will show below.

The numerical study of the regime $\delta<\pi$ requires care. As shown Fig. 1 (middle), the usual single-link update will not succeed in changing the monopole flux by $2 \pi$, and thus is not ergodic. To maintain ergodicity requires updating at least two links simultaneously, as shown Fig. 1 (right). 

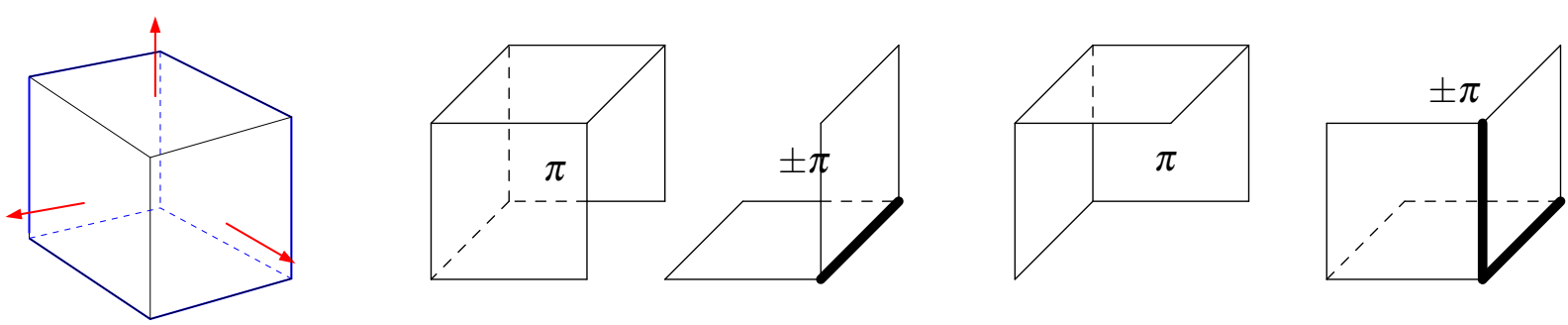

Figure 1: Left: A magnetic monopole is identified via its $2 \pi$ magnetic flux exiting an elementary cube. Thus, it requires some plaquette angles to reach or exceed $\pi / 3$. If the action forbids such plaquette angles, monopoles are absent. Middle: Creating or destroying a monopole requires changing the magnetic flux exiting an elementary cube by $2 \pi$. A $2 \pi$ flux change via a single-link update implies changing by $\pi$ the angles of two plaquettes. Thus, if the action forbids plaquette angles larger than $\pi$ and a single-link update is used, monopoles will be frozen and ergodicity will be lost. Right: This problem disappears, and ergodicity is restored, if two links are updated simultaneously.

\section{Helicity modulus and confining/Coulomb phase transition}

The helicity modulus $h$ measures the response of the system to a twist in the boundary conditions:

$$
\left.h \equiv \frac{\partial^{2} f\left(\phi_{\mu v}\right)}{\partial \phi_{\mu v}^{2}}\right|_{\phi_{\mu v}=0},
$$

where $f$ is the free energy density and $\phi_{\mu \nu}$ is an external flux through the $(\mu, v)$ planes. In a massive (confining) phase, $h$ goes to zero exponentially with the system size. In a massless (Coulomb) phase, it can be non-zero in the thermodynamic limit. Thus, it can be used as an order parameter in the $4 d U(1)$ theory, as shown in [10]. While $h$ can be expressed as a simple expectation value with the Wilson action, with the topological action it cannot. As in [7], we measured the distribution of allowed twist angles $\phi_{\mu v}$, fitting the curvature of the corresponding effective potential to obtain $h$.

In Fig. 2 (top), we show the measured values of the helicity modulus, as a function of $\beta$ for the Wilson action (left), and as a function of $\cos \delta$ for the topological action (right). The lines are fits assuming a first-order transition, where the free energy varies linearly with the control parameter (but with different coefficients in the two phases) [11]. The similarity between the two figures leaves no doubt that a first-order transition (confining $\leftrightarrow$ Coulomb) takes place with the topological action, at a cutoff angle $\cos \delta_{c} \approx-0.368$ quite different from $\delta=\pi / 3$. A measurement of Creutz ratios in both phases is consistent with an area law and a Coulomb law, respectively.

Note that the helicity modulus in the Coulomb phase has almost the same value at the transition for both actions. On the other hand, the fitted latent heat is about half as large with the topological action. Also, the monopole density changes discontinuously at the phase transition, for both actions, as illustrated Fig. 2 (bottom), but the topological action yields a smaller density and associated jump (right).

In addition to being an order parameter, the helicity modulus leads also to a simple definition of the renormalized charge [10]. The free energy $f\left(\phi_{\mu v}\right)$ is well described by the classical ansatz

$$
f\left(\phi_{\mu v}\right)=-\log \sum_{k} e^{-\frac{\beta_{R}}{2}\left(\phi_{\mu v}-2 \pi k\right)^{2}}
$$

from which $\beta_{R}=1 / e_{R}^{2}$ can be extracted, for either type of action. 

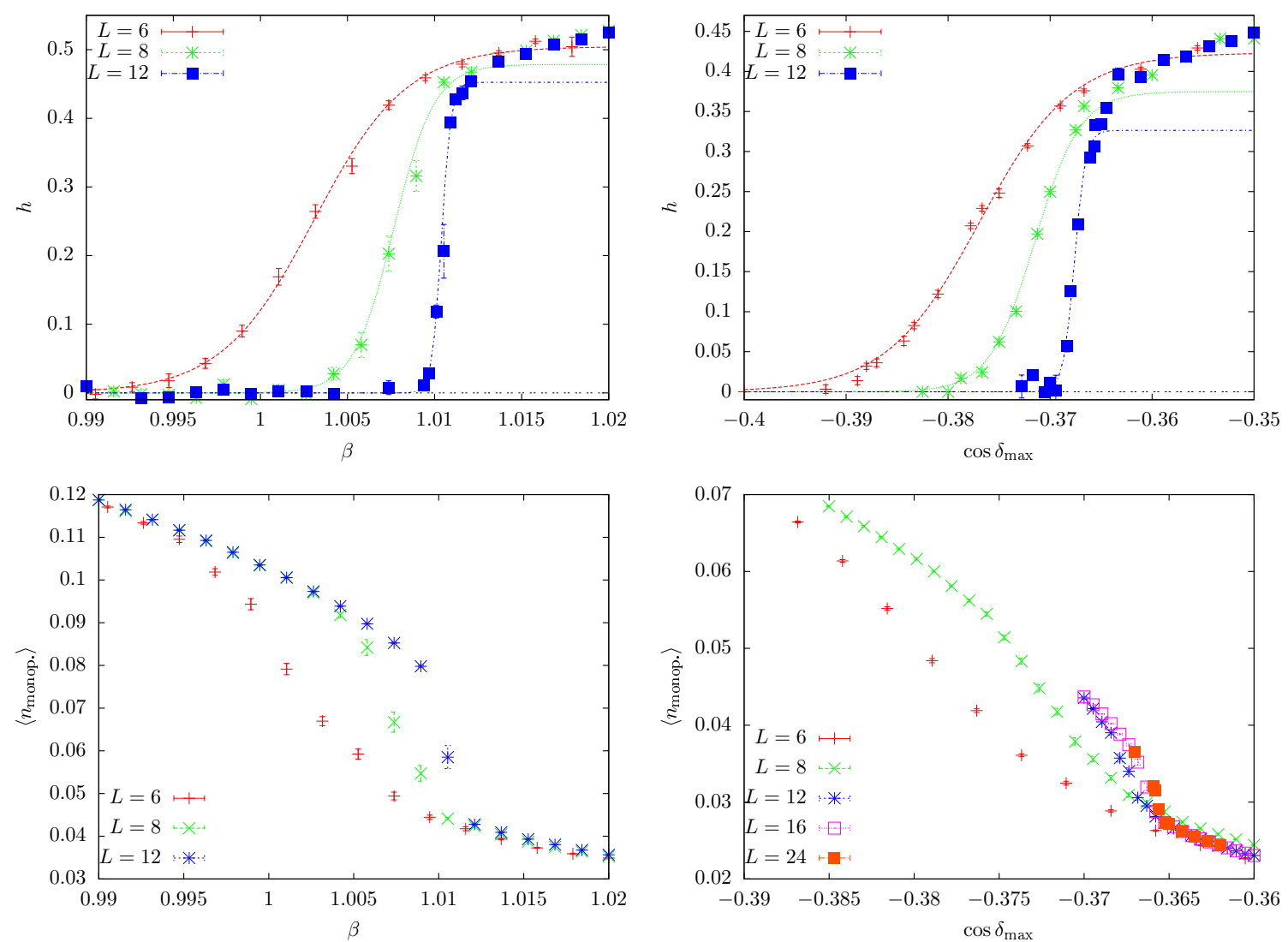

Figure 2: Helicity modulus (top row) and monopole density (bottom row) for the Wilson action (left column) and for the topological action (right column), across the phase transition. For both actions, the transition is first-order: the helicity modulus jumps at the transition and is well described by a finite-size first-order ansatz [11]; the monopole density also jumps, but the jump is smaller for the topological action.

\section{Monopole density in the Coulomb phase}

In the confining phase, monopoles condense. In the Coulomb phase, monopoles are exponentially suppressed: the density of monopole current behaves as $\exp \left(-\beta_{R} M\right)$, where $M$ can be considered as the monopole mass. Fig. 3 (left) shows this exponential suppression, for both actions. With the topological action, monopoles are much heavier, i.e. more suppressed. This is evidence that the topological action is improved: for a given value of the renormalized coupling, lattice artifacts are reduced considerably.

Actually, as explained in Sec. 2, with the topological action monopoles cannot exist if the cutoff $\delta$ on the plaquette angle is $\pi / 3$ or less. Thus, the density of monopole current vanishes, and is singular at $\delta=\pi / 3$. We tried to monitor this singular behavior. As $\delta$ approaches $\pi / 3$, the density of monopole current deviates from the exponential behavior of Fig. 3 (left) and seems to obey a power law, being proportional to $(\cos (\pi / 3)-\cos (\delta))^{\gamma}$. See Fig. 3 (right). We measure a large value $\sim 12$ for the "critical exponent" $\gamma$, which can be explained heuristically.

Consider first a $2 d X Y$ model, with topological action $\exp \left(-S_{\text {top }}\right)=\prod_{\langle i j\rangle} \Theta\left(\delta-\left|\theta_{i}-\theta_{j}\right|\right)$. Vortices in this model come from the spin orientation $\theta_{i}$ winding by $2 \pi$ as one goes around an 
elementary plaquette. Thus, vortices cannot exist if $\delta<\pi / 2$. When $\delta$ just exceeds $\pi / 2$, each angle $\left|\theta_{i}-\theta_{j}\right|$ around a vortex must be near $\pi / 2$, within an angle $(\delta-\pi / 2)$ of it. The phase space for this is $\propto(\delta-\pi / 2)$. Since there are 4 links around a vortex, related by one constraint (the sum of the four $\left(\theta_{i}-\theta_{j}\right)$ is $\left.2 \pi\right)$, the phase space for a vortex is $\propto(\delta-\pi / 2)^{3}$. Now, on a periodic lattice a vortex is always accompanied by an anti-vortex. Thus, the probability of finding a vortex anti-vortex pair should be $\propto(\delta-\pi / 2)^{6}$. This is remarkably consistent with the measurements shown Fig. 4 (right). We can then apply a similar argument to the $U(1)$ monopole density. The smallest monopole current loop is dual to 4 cubes. Each cube contains 6 plaquettes, which together obey a Bianchi constraint so that the total outgoing flux through the 6 plaquettes is $2 \pi$. Thus, the probability of finding such a monopole loop is $\propto(\delta-\pi / 3)^{4 \times 5}$. Our data, shown Fig. 4 (left), is more consistent with an exponent $\sim 12$, indicating that our heuristic argument is too crude.
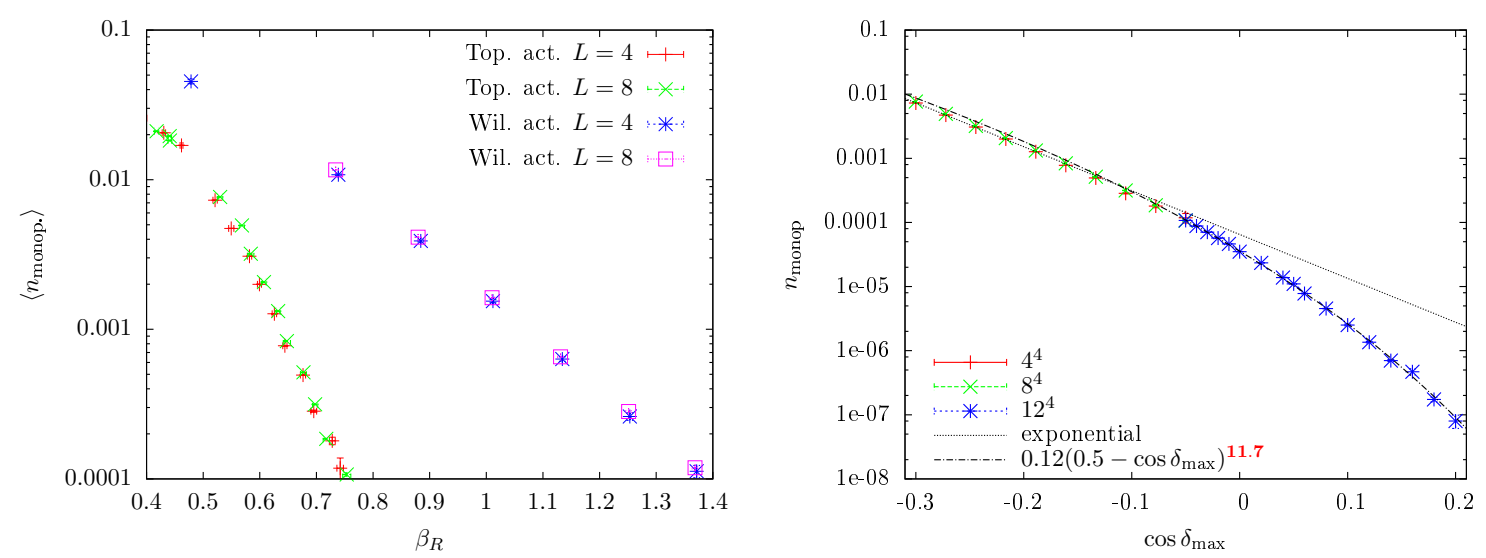

Figure 3: Monopole density in the Coulomb phase. Left: The monopole density decreases exponentially as a function of the inverse renormalized coupling $\beta_{R}=1 / e_{R}^{2}$, both for the Wilson action and for the topological action, with a steeper decrease for the latter. Right: using the topological action, the monopole density vanishes when the plaquette angles cannot exceed $\pi / 3$. As one approaches this critical value, the monopole density vanishes as a power law of the distance to the critical constraint angle, with a critical exponent $\sim 12$.

\section{Free energy for free}

With a topological action, the partition function $Z(\delta)$ simply counts the number of configurations which satisfy the constraint. Therefore, $Z\left(\delta_{2}\right) / Z\left(\delta_{1}\right)$, with $\delta_{2}<\delta_{1}$, is the proportion of configurations satisfying the constraint $\delta_{1}$ which also satisfy the tighter constraint $\delta_{2}$. These configurations can be counted as the Monte Carlo sampling of $Z\left(\delta_{1}\right)$ proceeds, giving the variation of the free energy $f(\delta)$ "for free".

We have applied this simple strategy near the phase transition, in the $2 d X Y$ model and in the $4 d U(1)$ gauge theory. The phase transition is of infinite order (BKT) in the former case, of first-order in the latter. The results for $\partial f(\delta) / \partial \delta$, shown Fig. 5, make this difference clear: for the first-order transition (left), the first derivative of the free energy develops a discontinuity as the system size increases, while it remains smooth for the infinite-order transition. 

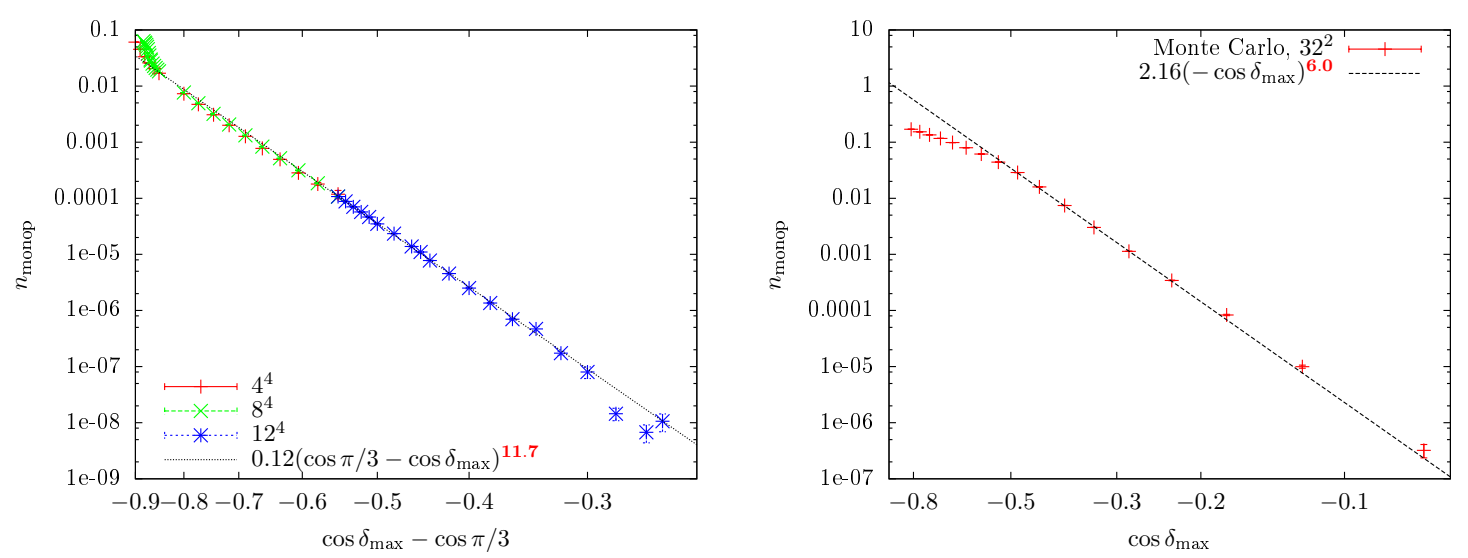

Figure 4: Left: monopole density in the Coulomb phase, for the topological action, as a function of the distance to the critical constraint: a power law is clearly visible on this log-log plot. Right: similar figure showing the density of vortices in the massless phase of the $2 d \mathrm{XY}$ model, using a topological action. The vortex density vanishes as a power of the distance to the critical constraint, which limits the difference among neighboring spin angles to $\pi / 2$. The critical exponent 6 is consistent with a heuristic argument (see text).
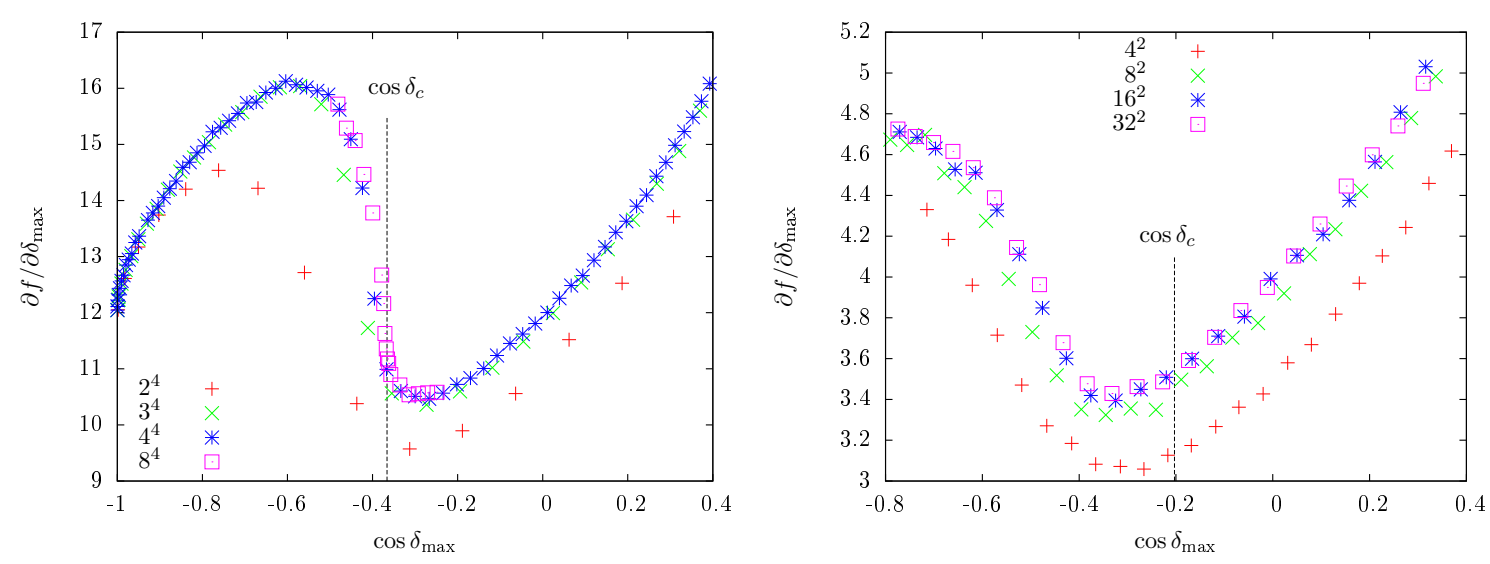

Figure 5: Derivative of the free energy density with respect to the constraint angle, across the confinementCoulomb phase transition marked by the vertical line, for the $4 d U(1)$ gauge theory (left) and for the $2 d X Y$ model $(r i g h t)$. As the system size is increased, a discontinuity is clearly visible in the former case, signaling a first-order transition. In contrast, the derivative of the free energy remains smooth in the $X Y$ model, as expected from a transition of infinite order. With a topological action, the free energy is obtained by simply counting the number of configurations which survive a tighter constraint (see text).

\section{Conclusion}

A topological action takes only two values: 0 and $+\infty$, where the latter removes any configuration which violates a local smoothness constraint. We have studied the properties of the topological action $S(\delta)=-\sum_{P} \log \Theta\left(\delta-\left|\theta_{P}\right|\right)$ in the $4 d U(1)$ gauge theory, as a function of the constraint angle $\delta$. As $\delta \rightarrow 0$, all plaquettes $P$ are forced to approach the identity. Yet the approach to the continuum action $\int d^{4} x F_{\mu \nu}^{2}$ is not clear a priori. Nevertheless, we have shown that the phase diagram of this lattice theory is very similar to that obtained with the Wilson action, with a confining, 
disordered phase at large $\delta$, separated from a Coulomb, ordered phase at small $\delta$ by a first-order transition. This transition is associated with a jump in the density of magnetic monopoles, which condense in the confining phase and are exponentially suppressed in the Coulomb phase. With the topological action, the monopole density is smaller in the Coulomb phase, which reflects the improvement of the action. The helicity modulus serves both as an order parameter for the phase transition, and as a renormalized coupling in the Coulomb phase.

A feature specific to the topological action is the total suppression of monopoles, or of topological defects in general, for a particular value of the constraint (here, $\delta_{c}=\pi / 3$ ). This threshold is analogous to Lüscher's "admissibility condition", which guarantees that topological sectors are distinct, even on the lattice [12]. Here, the monopole density is singular at $\delta_{c}$ : we observe that it decreases as a high power of $\left(\delta-\delta_{c}\right)$ when $\delta \rightarrow \delta_{c}^{+}$, then is identically zero for $\delta<\delta_{c}$. Other effects of this phase transition, if any, remain to be elucidated.

Finally, a topological action provides a simple means to measure the free energy, as the fraction of configurations in a given ensemble which continue to satisfy the constraint as this constraint is tightened. This might be useful in some attempts to tackle the sign problem, where the free energy must be convoluted with a complex phase factor [13].

\section{References}

[1] K. Symanzik, Nucl. Phys. B 226, 187 (1983).

[2] C. Michael and M. Teper, Nucl. Phys. B 305, 453 (1988).

[3] P. Hasenfratz and F. Niedermayer, Nucl. Phys. B 414, 785 (1994) [hep-lat/9308004].

[4] J. Fingberg, U. M. Heller and V. K. Mitrjushkin, Nucl. Phys. B 435, 311 (1995) [hep-lat/9407011].

[5] J. Balog, F. Niedermayer, M. Pepe, P. Weisz and U.-J. Wiese, JHEP 1211, 140 (2012) [arXiv:1208.6232 [hep-lat]].

[6] D. Banerjee, M. Bögli, K. Holland, F. Niedermayer, M. Pepe, U. Wenger and U. J. Wiese, arXiv:1512.04984 [hep-lat].

[7] W. Bietenholz, M. Bögli, F. Niedermayer, M. Pepe, F. G. Rejon-Barrera and U. J. Wiese, JHEP 1303, 141 (2013) [arXiv:1212.0579 [hep-lat]].

[8] O. Akerlund and P. de Forcrand, JHEP 1506, 183 (2015) [arXiv:1505.02666 [hep-lat]].

[9] T. A. DeGrand and D. Toussaint, Phys. Rev. D 22, 2478 (1980).

[10] M. Vettorazzo and P. de Forcrand, Nucl. Phys. B 686, 85 (2004) [hep-lat/0311006].

[11] C. Borgs and R. Kotecky, Phys. Rev. Lett. 68, 1734 (1992).

[12] M. Luscher, Commun. Math. Phys. 85, 39 (1982).

[13] K. Langfeld and B. Lucini, Phys. Rev. D 90, no.9, 094502 (2014) [arXiv:1404.7187 [hep-lat]]. 